\title{
Influencia del mallado en el modelado computacional de incendios en centrales nucleares
}

\section{Mesh influence on the fire computer modeling in nuclear power plants}

\author{
D. Lázaro $^{(*)}$, M. Lázaro(*) ${ }^{(*}$. Peco ${ }^{(* *)}$, D. Alvear ${ }^{(*)}$
}

\section{RESUMEN}

Los modelos computacionales de incendio permiten estudiar las consecuencias de los incendios en escenarios reales. Su uso para el análisis y la mejora de la seguridad contra incendios en centrales nucleares se ha visto incrementado al publicarse la normativa que permite el empleo de métodos informados por el riesgo y basados en prestaciones. La selección del tamaño de celda es fundamental en estos modelos. El mallado debe establecer un compromiso entre su ajuste a la geometría, la resolución de las ecuaciones y los tiempos de cómputo. El presente artículo estudia distintos tamaños de celda mediante la herramienta FDS, con el objetivo de evaluar su influencia en los resultados simulados. Como punto de referencia se emplearon escenarios de interés en centrales nucleares. Los resultados ofrecen datos relevantes para los usuarios, mostrando los tamaños de celda óptimos para garantizar la calidad de las simulaciones y reducir las incertidumbres en sus resultados.

Palabras clave: modelado CFD, incendios en recintos cerrados, centrales nucleares, precisión de los resultados.

\section{ABSTRACT}

Fire computer models allow to study real fire scenarios consequences. Its use in nuclear power plants has increased with the new regulations to apply risk informed performance-based methods for the analysis and design of fire safety solutions. The selection of the cell side factor is very important in these kinds of models. The mesh must establish a compromise between the geometry adjustment, the resolution of the equations and the computation times. This paper aims to study the impact of several cell sizes, using the fire computer model FDS, to evaluate the relative affectation in the final simulation results. In order to validate that, we have employed several scenarios of interest for nuclear power plants. Conclusions offer relevant data for users and show some cell sizes that can be selected to guarantee the quality of the simulations and reduce the results uncertainty.

Keywords: CFD fire models, enclosure fire dynamics, nuclear power plants, results accuracy.

(*) Universidad de Cantabria (Santander, España).

(**) Consejo de Seguridad Nuclear (Madrid, España).

Persona de contacto/Corresponding author: lazarod@unican.es (D. Lázaro)

ORCID: http://orcid.org/oooo-0002-8150-4838. (D. Lázaro); http://orcid.org/oooo-00o2-1008-694X. (M. Lázaro); http://orcid.org/oooo-0002-3649-0246 (J. Peco); http://orcid.org/oooo-0002-7105-5282 (D. Alvear).

Cómo citar este artículo/Citation: Lázaro, D., Lázaro, M., Peco, J., Alvear, D. (2018). Influencia del mallado en el modelado computacional de incendios en centrales nucleares. Informes de la Construcción, 70(549): e238. https://doi.org/10.3989/id.55345

Copyright: (C) 2018 CSIC. Este es un artículo de acceso abierto distribuido bajo los términos de la licencia de uso y distribución Creative Commons Reconocimiento 4.0 Internacional (CC BY 4.0). 


\section{INTRODUCCIÓN}

A lo largo de la historia de las centrales nucleares, se comprueba que una gran parte de sus incidentes tienen su origen en el desarrollo y propagación de incendios. Uno de los casos más conocidos fue el de la central nuclear de Browns Ferry (Estados Unidos) (1), que supuso un hito muy importante en el desarrollo de medidas de protección contra incendios en centrales nucleares.

Fruto de esta y otras experiencias que se han ido recogiendo de diferentes incidentes, se fueron planteando modificaciones y mejoras en las normativas con el objetivo final de mejorar la seguridad contra incendios en las centrales nucleares. Con este propósito, en los últimos tiempos se editaron normas, como la NFPA 805 (2), mediante las cuales se utiliza una metodología informada por el riesgo y basada en prestaciones para, manteniendo los mismos estándares de seguridad contra incendios de la normativa convencional, implantar programas de protección contra incendios más eficientes que no precisen de unos márgenes de seguridad que, en ocasiones, podrían resultar demasiado conservadores. En Estados Unidos, la Nuclear Regulatory Commission (NRC) reconoce esta última norma como una opción a la que acogerse de forma voluntaria, que sustituiría a la tradicionalmente utilizada hasta ahora, y sobre la que basaría el programa de protección contra incendios para las plantas de energía nuclear que así lo decidieran, y paulatinamente estos métodos están ganando más fuerza. En España el Consejo de Seguridad Nuclear (CSN), a través de la Instrucción IS-30 (3), abrió también el camino a esta nueva vía de diseño del programa de protección contra incendios atendiendo una metodología informada por el riesgo y basada en prestaciones.

Igualmente, este tipo de diseño basado en prestaciones ha tenido un gran auge en los últimos años, permitiendo mejorar la seguridad de otras edificaciones (edificios de gran altura, grandes centros comerciales, estaciones de transporte intermodales, edificaciones singulares, etc.) (4).

Sin embargo, estos enfoques requieren un profundo conocimiento científico-técnico del fenómeno que se analiza y de las herramientas empleadas para su estudio, investigar sobre los posibles riesgos de incendio que pudiesen suceder y cuál pudiera ser la mejor solución para solventarlos y conocer en profundidad las características concretas de la edificación de estudio.

De entre esas herramientas para la simulación computacional de incendios, para este trabajo fue seleccionado el modelo 'Fire Dynamics Simulator, FDSv6' (5) del National Institute of Standards and Technology (NIST) (USA) que resuelve numéricamente las ecuaciones de Navier-Stokes, apropiadas para los flujos de baja velocidad, con especial énfasis en el humo y el transporte de calor generado durante los incendios. Además, es el modelo más empleado a nivel internacional y cuenta con cientos de validaciones en escenarios muy diversos, existiendo para el caso de las centrales nucleares un amplio trabajo de validación ayudado por una intensa labor experimental desarrollada por la NRC (6) (7).

Sin embargo, su empleo no es trivial, siendo necesario establecer unas bases y una metodología para su aplicación, así como definir el tamaño de malla, los parámetros característicos de los escenarios de incendios en los que se va a aplicar dentro de la central nuclear, la definición de la química de la reacción, las propiedades termodinámicas de los materiales disponibles o la ventilación disponible (8) (9) (10). En concreto, tal y como se indica en NUREG-1824 (11), el tamaño de malla es el parámetro numérico de mayor importancia en FDS.

En este contexto, el presente artículo analiza, a modo de análisis de sensibilidad, el impacto del tamaño de la celda empleada para definir el dominio computacional, con el objetivo de evaluar y definir la influencia del mallado del modelo en los resultados finales de la simulación y que pueda servir de guía para el uso adecuado del modelo en esta clase de entornos.

En primer lugar, se presentará la metodología seguida para seleccionar los tamaños de celda recomendados para el modelado de los distintos escenarios en FDS. Seguidamente se presentan la definición y resultados de los escenarios empleados en el estudio. De esta forma, se incluye el escenario de pequeña escala denominado «Penlight» (CAROLFIRE (12)). Seguidamente, se incluye el análisis en una sala de interruptores para estudiar el efecto en escenarios a gran escala.

\section{METODOLOGÍA}

Uno de los aspectos fundamentales a la hora de definir un escenario de incendios con un modelo de fluidodinámica computacional de incendios - CFD como FDS es la selección de un tamaño de celda adecuado. Este ha de ser lo suficientemente pequeño para que permita resolver las ecuaciones de turbulencia, pero también lo suficientemente grande como para no incurrir en un coste computacional inasumible.

Este tamaño va a determinar los volúmenes de las celdas empleadas para resolver las ecuaciones de conservación de masa, momento y energía, teniendo cada celda las mismas propiedades de temperatura, velocidad, concentración de gases, etc. Es por ello que el error asociado con la discretización de las derivadas parciales depende del tamaño de la celda empleado.

No existe una regla general que defina el tamaño más óptimo a elegir, ya que esta depende del caso concreto estudiado, y muchas veces es la experiencia del usuario la que hace que se produzca una u otra selección, sin embargo, se pueden emplear algunos métodos para ayudar a esa selección, como realizar un análisis de sensibilidad realizando varias simulaciones y reduciendo el tamaño de celda en cada una de ellas hasta alcanzar una convergencia, o emplear indicadores como el diámetro característico del incendio. FDS (13) plantea el uso de ese diámetro característico del incendio para tener una estimación de ese valor. Este cálculo está focalizado para escenarios en los cuales se estudie el movimiento de humos. Según este criterio el cociente entre el tamaño de celda característico, $\Delta \mathrm{z}$, y el diámetro característico del fuego, $\mathrm{D}^{*}$ [1], es un factor que permite seleccionar el tamaño de celda, y habitualmente $D^{*} / \Delta \mathrm{z}$ se recomienda que esté entre valores de 4 y 16 (11) para que proporcione resultados satisfactorios.

$$
D^{*}=\left(\frac{\dot{Q}}{\rho c_{p} T \sqrt{g}}\right)^{2 / 5}
$$

Donde es la velocidad de cesión de calor $(\mathrm{kW})$, la densidad $\left(\mathrm{kg} / \mathrm{m}^{3}\right)$, el calor específico $(\mathrm{J} / \mathrm{kgK}), T$ la temperatura ambiente, y $g$ la aceleración de la gravedad $\left(\mathrm{m} / \mathrm{s}^{2}\right)$. 
Esta metodología se ha aplicado al segundo escenario, en el cual el estudio del movimiento de humos es de gran importancia. Mediante la simulación de una sala de interruptores se ha estudiado la influencia del tamaño de la celda en escenarios a gran escala. Se han seleccionado tamaños de celdas basados en las recomendaciones del manual de usuario de FDS, de modo que se incluyan tanto valores de $\mathrm{D}^{*} /$ $\Delta \mathrm{z}$ recomendados en FDS como alguno otro fuera de estas recomendaciones con objeto de estudiar su efecto en los resultados. En cuanto al primer escenario, nos permite evaluar el efecto del tamaño de celda en la transferencia térmica considerando y sin considerar la resolución de las reacciones de degradación de los cables.

Para analizar la diferencia en los resultados de los distintos casos simulados para cada escenario, se ha considerado el error definido por la ecuación [3].

$$
\begin{aligned}
\operatorname{MSE}\left(T_{i}\right) & =\sqrt{\frac{\sum_{t}\left(T_{i}(t)-T_{\text {base }}(t)\right)^{2}}{(N-1)}} \\
\varepsilon & =\frac{\operatorname{MSE}\left(T_{i}\right)}{\bar{T}_{i}}
\end{aligned}
$$

donde es la temperatura del caso $i$ en el instante de tiempo $t, N$ es el número de valores de temperatura para cada caso, es la temperatura del caso empleado como referencia en el instante de tiempo $t$ y es la temperatura media del caso $i$. $\mathrm{El}$ equipo empleado en todas las simulaciones es un 2 Xeon X5550@2.67GHz (16 núcleos) de 32 GB con un sistema Ubuntu 14.04.2 LTS.

\section{RESULTADOS}

\subsection{Escenario «Penlight»}

Dentro del proyecto CAROLFIRE (12) se realizaron un total de 78 ensayos a pequeña escala en las instalaciones del Sandia National Laboratories (USA), recibiendo el escenario ensayado el nombre de «Penlight». Estos ensayos permitieron estudiar el fallo de diferentes tipos de cables al ser sometidos a distintos flujos de temperatura, consistiendo el ensayo en un horno cilíndrico en cuyo eje se sitúan los distintos tipos de cable que son sometidos a un flujo de calor por radiación. Como muestra la Figura 1, el horno es un cilindro de unos 80 cm de largo con diámetro de $51 \mathrm{~cm}$ en el interior del cual se colocan dos cables, uno para monitorización térmica y otro para monitorización eléctrica. De este modo todo el cable queda expuesto a la radiación proveniente de las paredes del horno. Dado que la importancia del estudio se centra en el análisis de la malla en función de los resultados de temperatura obtenidos en los cables, se ha reducido el tamaño del dominio computacional empleado a $0.6 \mathrm{~m} \times 0.45 \mathrm{~m} \times 0.45 \mathrm{~m}$, manteniendo el flujo de calor por radiación aplicado sobre los cables $\left(87 \mathrm{~kW} / \mathrm{m}^{2}\right)$.

Se midió la temperatura del cable a una profundidad de 2 $\mathrm{mm}$, y para realizar el análisis de sensibilidad de la rejilla se analizaron dos tipos diferentes de cables, uno inerte, con las instrucciones PART o con OBST de FDS para ver la influencia de la rejilla en cada caso, y seguidamente, otro cable con propiedades reactivas. La instrucción OBST de FDS se emplea para definir cualquier tipo de geometría. Generalmente deberá ajustarse al tamaño de la celda, pero podrá indicársele espesores mayores o menores que el tamaño de la celda. Por su parte, la instrucción PART se emplea generalmente para objetos especialmente pequeños y se resuelve con el tamaño de celda definido, soliéndose emplear para gotas, partículas y en algunos casos para cables.

\section{Cables inertes}

Se definieron unos cables inertes con una densidad de 1959 $\mathrm{kg} / \mathrm{m}^{3}$, una conductividad térmica de $0.2 \mathrm{~W} / \mathrm{mK}$ y un calor específico de $500 \mathrm{~J} / \mathrm{kg} \mathrm{K}$. La Tabla 1 muestra los distintos escenarios simulados, considerando tanto la variación del tamaño de celda como del método de introducción del cable.

Los resultados de temperatura obtenida en los distintos casos estudiados se muestran en la Figura 2.

La Tabla 2 muestra la variación de los resultados de la temperatura obtenidos a través del cálculo del error (), en base a dos criterios. En primer lugar, la segunda columna de la tabla muestra la variación considerando como caso base la simulación con el menor tamaño de celda $(2.5 \mathrm{~cm})$. La tercera

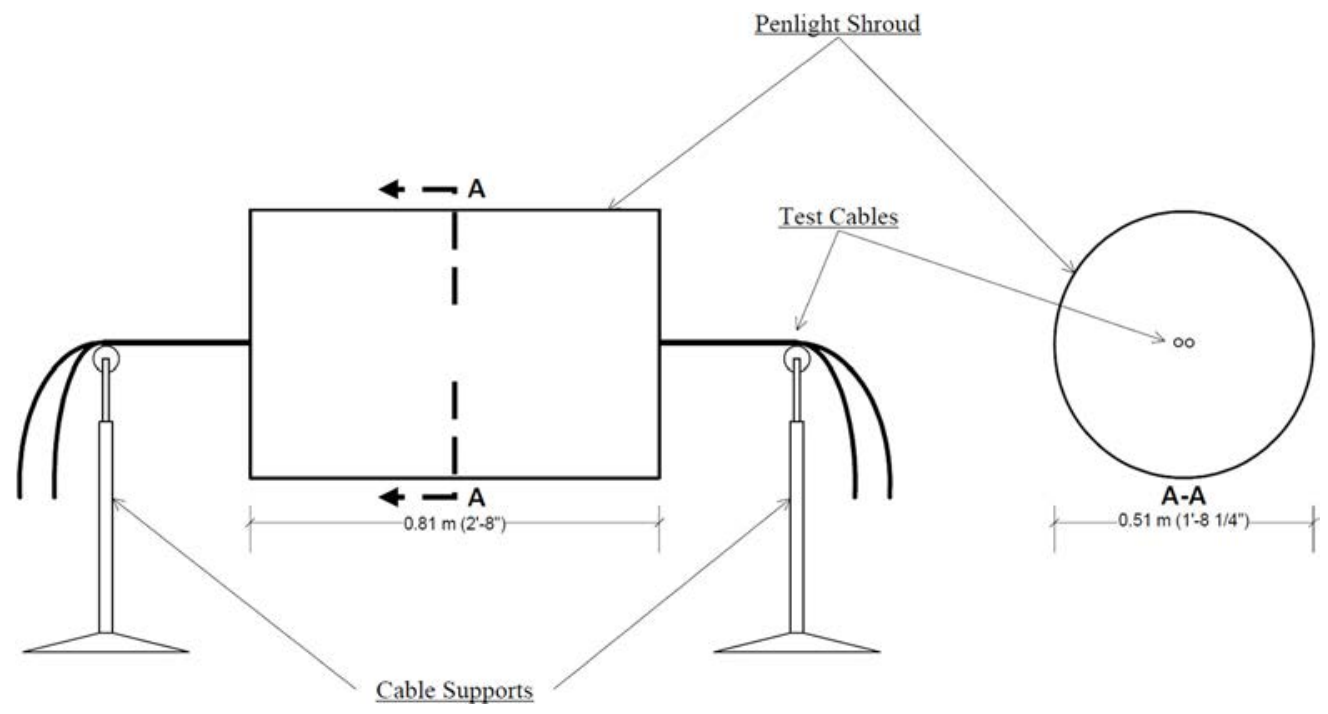

Figura 1. Esquema del ensayo Penlight en configuración air drop (12). 
Tabla1. Casos estudiados para el cable inerte.

\begin{tabular}{|c|c|c|c|}
\hline Caso & $\mathbf{N}^{\mathbf{0}}$ celdas & Tamaño (forma) & Instrucción \\
\hline $\mathbf{1}$ & 7776 & $2.5 \mathrm{~cm}$ (cúbica) & PART \\
\hline $\mathbf{2}$ & 972 & $5 \mathrm{~cm}$ (cúbica) & PART \\
\hline $\mathbf{3}$ & 150 & $\begin{array}{c}10 \mathrm{~cm}, 9 \mathrm{~cm}, 9 \mathrm{~cm} \\
\text { (paralelepípeda) }\end{array}$ & PART \\
\hline $\mathbf{4}$ & 972 & $5 \mathrm{~cm}$ (cúbica) & OBST \\
\hline $\mathbf{5}$ & 150 & $\begin{array}{c}10 \mathrm{~cm}, 9 \mathrm{~cm}, 9 \mathrm{~cm} \\
\text { (paralelepípeda) }\end{array}$ & OBST \\
\hline
\end{tabular}

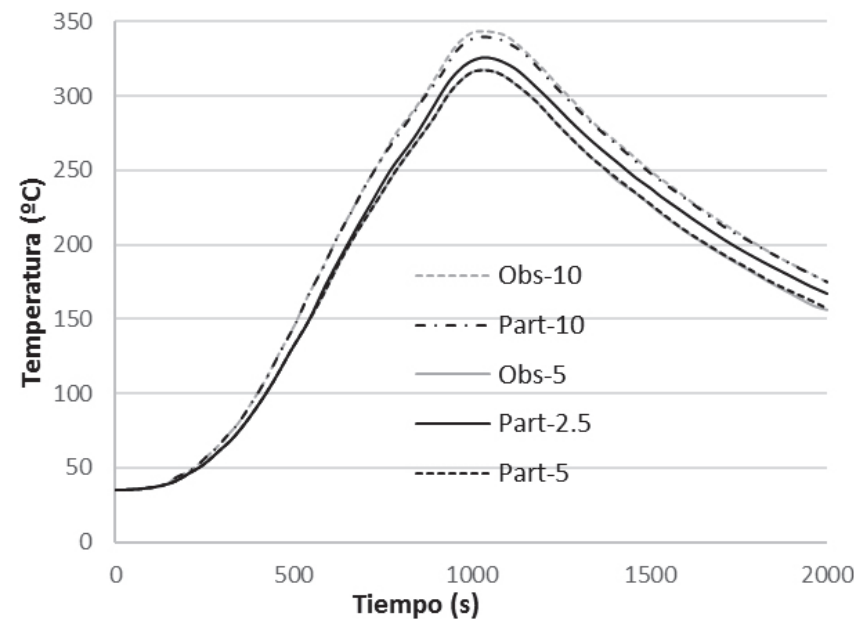

Figura 2. Temperatura del cable inerte en los casos simulados.

columna muestra la variación de la temperatura atendiendo únicamente al método de introducción del cable empleado.

Se observa como para distintos tamaños de celda hay una variación de en torno al $4 \%$ si consideramos un recinto con 8 veces menos celdas y en torno al $6 \%$ si reducimos el número de celdas en el recinto de 7776 hasta 150. En lo que respecta a la variación del método de introducción del cable, no se han observado grandes variaciones, estando en los dos casos estudiados por debajo del $1 \%$ de variación.

\section{Cables con propiedades reactivas}

Para estudiar la influencia de la malla cuando tienen lugar reacciones de descomposición de fase sólida se han considerado en el estudio cables de PVC con propiedades reactivas. La Tabla 3 recoge las propiedades introducidas en el modelo para definir los cables estudiados.

La temperatura de referencia indica la temperatura a la cual tiene lugar la reacción de descomposición del PVC, que necesita una energía de $1500 \mathrm{~J} / \mathrm{kg}$ desencadenarse. El calor de combustión es la energía que este libera al reaccionar el gas liberado en la descomposición al reaccionar con el oxígeno del ambiente.
Tabla 2. Variación de la temperatura en los cables inertes.

\begin{tabular}{|c|c|c|}
\hline Caso & & OBSTvsPART \\
\hline $\mathbf{1}$ & BASE & - \\
\hline $\mathbf{2}$ & $3.97 \%$ & BASE \\
\hline $\mathbf{3}$ & $5.67 \%$ & BASE \\
\hline $\mathbf{4}$ & $4.25 \%$ & $0.57 \%$ \\
\hline $\mathbf{5}$ & $6.38 \%$ & $0.99 \%$ \\
\hline
\end{tabular}

La Tabla 4 recoge los casos simulados para realizar el análisis de la influencia del tamaño de celda y del método de introducción del cable en FDS.

La Figura 3 muestra la temperatura en los distintos casos simulados. Se puede observar claramente la desviación de las curvas de temperatura en los casos 3 y 4 correspondientes a las simulaciones con mayor tamaño de celda.

En la Tabla 5 se observa la variación de los resultados de la temperatura para las distintas simulaciones. La segunda columna muestra la variación de la temperatura tomando como caso base las simulaciones con el menor tamaño de celda. Se puede ver cómo para la definición de los cables mediante las instrucciones PART y OBST se obtiene la misma variación con el mayor tamaño de celda con respecto al menor (en torno al 12.5\%). Sin embargo, con el tamaño mediano de celda se obtienen distintas variaciones en la temperatura del cable dependiendo del tipo de instrucción empleada para definir el cable.

La tercera columna muestra la variación de la temperatura en el cable en función de la instrucción empleada para simular el mismo. Se observa como en este escenario, en el que los cables tienen propiedades reactivas, se produce una variación del 3.18 \% en los casos con los tamaños de celda menores. Para tamaños de celda mayores la variación no alcanza el 0.3\%.

Vemos como, una variación del tamaño de celda de $2.5 \mathrm{~cm}$ a 5 $\mathrm{cm}$ supone variaciones en la temperatura inferiores al $5.39 \%$ en todos los casos. Sin embargo, dependiendo del dominio computacional requerido, el empleo de celdas de $2.5 \mathrm{~cm}$ puede ser computacionalmente inviable.

\subsection{Sala de interruptores}

Las salas de interruptores son típicas en las centrales nucleares y contienen en su interior una gran cantidad de bandejas de cables, cabinas eléctricas y conductos de ventilación (14). El recinto considerado dispone en su interior de 16 bandejas de cables, 12 cabinas eléctricas, 3 salidas de aire de $1 \mathrm{~m} 2$ cada una, una rejilla de entrada de aire con un flujo de $1.73 \mathrm{~m} 3 / \mathrm{s}$ y una fuente de ignición de $2 \mathrm{MW}$ situada a $2 \mathrm{~m}$ de altura. El recinto tiene un tamaño de $17 \mathrm{~m}$ de longitud, $10 \mathrm{~m}$ de ancho y 4.6 de alto.

Tabla 3. Propiedades de los cables de PVC.

\begin{tabular}{|l|c|c|c|}
\hline \multicolumn{1}{|c|}{ Propiedades } & Valor & Propiedades & Valor \\
\hline Densidad & $1200 \mathrm{~kg} / \mathrm{m}^{3}$ & Nu_Spec & 1 \\
\hline Calor específico & $1370 \mathrm{~J} / \mathrm{kg} . \mathrm{K}$ & Spec_Id & PVC \\
\hline Conductividad & $0.12 \mathrm{~W} / \mathrm{m} . \mathrm{K}$ & Temperatura de referencia & $257^{\circ} \mathrm{C}$ \\
\hline Emisividad & 0.21 & Calor de reacción & $1500 \mathrm{~J} / \mathrm{kg}$ \\
\hline $\mathrm{N}^{\mathrm{O}}$ Reacciones & 1 & Calor de combustión del PVC & $16400 \mathrm{~J} / \mathrm{kg}$ \\
\hline
\end{tabular}


Tabla 4. Casos estudiados para el cable con propiedades reactivas.

\begin{tabular}{|c|c|c|c|}
\hline Caso & No celdas & Tamaño (forma) & Instrucción \\
\hline $\mathbf{1}$ & 7776 & $2.5 \mathrm{~cm}$ (cúbica) & PART \\
\hline $\mathbf{2}$ & 972 & $5 \mathrm{~cm}$ (cúbica) & PART \\
\hline $\mathbf{3}$ & 150 & $\begin{array}{c}10 \mathrm{~cm}, 9 \mathrm{~cm}, 9 \mathrm{~cm} \\
\text { (paralelepipedo) }\end{array}$ & PART \\
\hline $\mathbf{4}$ & 7776 & $2.5 \mathrm{~cm}$ (cúbica) & OBST \\
\hline $\mathbf{5}$ & 972 & $5 \mathrm{~cm}$ (cúbica) & OBST \\
\hline $\mathbf{6}$ & 150 & $\begin{array}{c}10 \mathrm{~cm}, 9 \mathrm{~cm}, 9 \mathrm{~cm} \\
\text { (paralelepipedo) }\end{array}$ & OBST \\
\hline
\end{tabular}

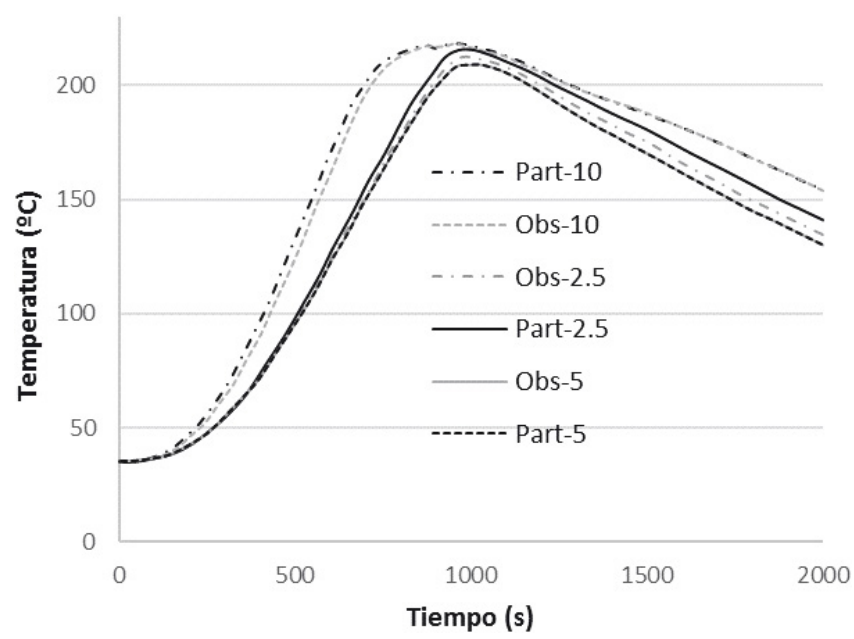

Figura 3. Temperatura en los cables de los casos simulados.

La Figura 4 muestra los puntos de medida de la temperatura que van a emplearse en el análisis de los distintos tamaños de celda. Se va a estudiar la temperatura del gas y la temperatura a $2 \mathrm{~mm}$ de profundidad de los cables mediante termopares posicionados en la vertical de la fuente de ignición $(x=2.6 \mathrm{~m}$, $\mathrm{y}=1.9 \mathrm{~m}, \mathrm{z}=2.9 \mathrm{~m}$ para la medición de la temperatura del gas, y $\mathrm{x}=2.6 \mathrm{~m}, \mathrm{y}=1.9 \mathrm{~m}, \mathrm{z}=3.2 \mathrm{~m}$ para la medición de la temperatura del cable) y en una zona no afectada directamente por el penacho $(x=9.6 \mathrm{~m}, \mathrm{y}=7.4 \mathrm{~m}, \mathrm{z}=2.9 \mathrm{~m}$ para la medición de la temperatura del gas, $\mathrm{y} x=9.6 \mathrm{~m}, \mathrm{y}=7.4 \mathrm{~m}, \mathrm{z}=3.2 \mathrm{~m}$ para la medición de la temperatura del cable).
Tabla 5. Variación de la temperatura en los casos simulados.

\begin{tabular}{|c|c|c|}
\hline Caso & & OBSTvsPART \\
\hline $\mathbf{1}$ & BASE & BASE \\
\hline $\mathbf{2}$ & $5.39 \%$ & BASE \\
\hline $\mathbf{3}$ & $12.33 \%$ & BASE \\
\hline $\mathbf{4}$ & BASE & $3.18 \%$ \\
\hline $\mathbf{5}$ & $2.27 \%$ & $0.24 \%$ \\
\hline $\mathbf{6}$ & $12.76 \%$ & $0.28 \%$ \\
\hline
\end{tabular}

Se han seleccionado puntos de medida de la temperatura en la fase gas y la fase sólida y sobre el penacho del incendio y en una zona alejada para poder estudiar los distintos casos de medición de temperaturas en un recinto singular de incendios. Todas las bandejas de cables tienen en su parte inferior una estructura metálica. Dado que todas las simulaciones han sido realizadas bajo las mismas condiciones de contorno (ventilación, propiedades de los materiales, posición de los puntos de medida), estos parámetros no van a afectar a la comparación del tamaño de celdas.

La Tabla 6 muestra los 4 casos estudiados. Se han empleado tamaños de malla de $0.2 \mathrm{~m}, 0.15 \mathrm{~m}, 0.1 \mathrm{~m}$ y $0.05 \mathrm{~m}$, considerando este último como el base a la hora de calcular los errores. Con esto se han conseguido unos valores de $D^{*} / \Delta z$ [1] entre 6 y 26.

La Tabla 7 muestra los errores calculados con la ecuación [3] para los distintos puntos de medida de la temperatura.

Los resultados muestran como a menor tamaño de celda es menor el error obtenido, siendo casi 16 veces mayor el error en el caso 1 con respecto al 3 para la temperatura del cable bajo el penacho. Además, se observa que, debido a las mayores temperaturas y a las mayores turbulencias a resolver por el modelo, los errores son mayores en la zona del penacho, justo encima de la fuente de ignición. La diferencia de la temperatura entre el caso 4, con el tamaño de celda de $0.05 \mathrm{~m}$, y el caso 3 con un tamaño de celda de $0.1 \mathrm{~m}$, es del $8.77 \%$ en el cable sobre el penacho. Sin embargo, para
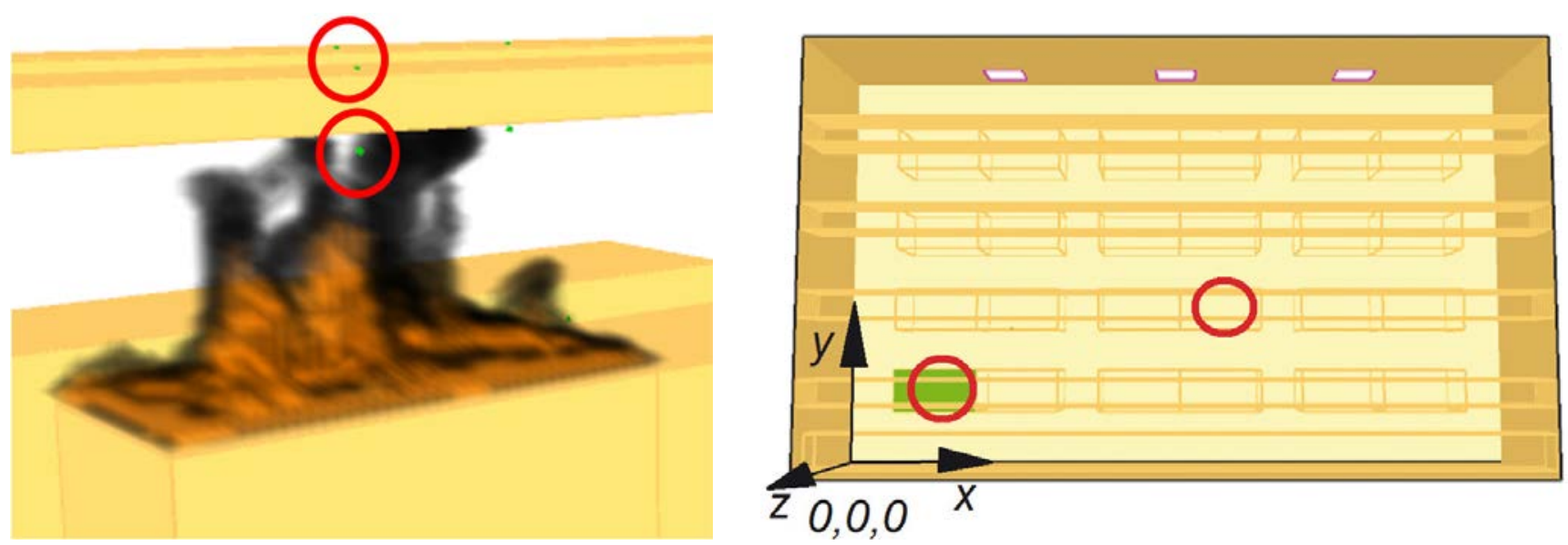

Figura 4. Fuente de incendio sobre una cabina eléctrica (izquierda), vista en planta de la sala de interruptores (derecha). 
Tabla 6. Características de la malla y tiempo de simulación.

\begin{tabular}{|c|c|c|c|c|}
\hline Caso & $\boldsymbol{D}^{*} / \boldsymbol{\Delta z}$ & $\begin{array}{c}\text { Tamaño } \\
\text { celda }\end{array}$ & $\mathbf{N}^{\mathbf{o}}$ celdas & $\begin{array}{c}\text { Tiempo } \\
\text { cómputo }\end{array}$ \\
\hline $\mathbf{1}$ & 6.3 & $20 \mathrm{~cm}$ & 97750 & $4 \mathrm{~h} 27^{\prime}$ \\
\hline $\mathbf{2}$ & 8.4 & $15 \mathrm{~cm}$ & 235600 & $11 \mathrm{~h} 42^{\prime}$ \\
\hline $\mathbf{3}$ & 12.6 & $10 \mathrm{~cm}$ & 782000 & $36 \mathrm{ho9}$ \\
\hline $\mathbf{4}$ & 25.3 & $5 \mathrm{~cm}$ & 6256000 & $428 \mathrm{~h} 47^{\prime}$ \\
\hline
\end{tabular}

el resto de los puntos de control el error está por debajo del $2.26 \%$, mientras que el tiempo de cómputo es 11.86 veces inferior.

\section{CONCLUSIONES}

El presente artículo presenta un análisis de la influencia del tamaño de las celdas del mallado en los resultados del modelo de simulación computacional FDS en un ensayo de cables a pequeña escala y de una sala de interruptores típica de una central nuclear.

En el análisis del mallado se han estudiado algunos de los elementos más característicos para la propagación de incendios en centrales nucleares, como son las bandejas de cables y los paneles eléctricos locales. Hemos podido constatar como en un escenario a pequeña escala como el ensayo «Penlight», aumentando el tamaño de celda de $2.5 \mathrm{~cm}$ de lado hasta $5 \mathrm{~cm}$ se obtienen diferencias en los valores de temperatura de hasta un $5.39 \%$, siendo de hasta el $12.76 \%$ cuando se utilizan tamaños de celda de $10 \mathrm{~cm}$ de lado.

Sin embargo, en ciertas ocasiones tamaños de celda de $5 \mathrm{~cm}$ de lado o menores son computacionalmente inviables debido a los tiempos de cómputo requeridos para su procesamiento. Por ello, se ha considerado el estudio de un escenario de ma-
Tabla 7. Resultados para la sala de control.

\begin{tabular}{|c|c|c|c|c|}
\hline Caso & $\begin{array}{c}\text { gas } \\
\text { penacho }\end{array}$ & $\begin{array}{c}\text { cable } \\
\text { penacho }\end{array}$ & gas & cable \\
\hline $\mathbf{1}$ & $24.61 \%$ & $24.89 \%$ & $6.65 \%$ & $14.14 \%$ \\
\hline $\mathbf{2}$ & $13.72 \%$ & $15.98 \%$ & $5.37 \%$ & $10.75 \%$ \\
\hline $\mathbf{3}$ & $8.77 \%$ & $1.56 \%$ & $2.26 \%$ & $1.84 \%$ \\
\hline $\mathbf{4}$ & $\mathrm{BASE}$ & $\mathrm{BASE}$ & $\mathrm{BASE}$ & $\mathrm{BASE}$ \\
\hline
\end{tabular}

yor escala, obteniendo como resultado una diferencia máxima de temperaturas del $8.77 \%$ cuando variamos el tamaño de celda de $5 \mathrm{~cm}$ a $10 \mathrm{~cm}$ de lado. Empleando tamaños de celda de $15 \mathrm{~cm}$ y de $20 \mathrm{~cm}$ se obtienen diferencias de hasta el $15.98 \%$ y el $24.89 \%$ respectivamente. Se ha observado que el tamaño de celda afecta principalmente a la temperatura en la vertical del penacho y a la temperatura de elementos sólidos como, por ejemplo, los cables.

Como conclusión final se recomienda que, para escenarios de fuego en centrales nucleares de características semejantes a los analizados en el presente trabajo, se empleen tamaños de celda de $5 \mathrm{~cm}$ cuando sean escenarios de pequeña escala (alrededor de $0.12 \mathrm{~m} 3$ ), y de $10 \mathrm{~cm}$ en el caso de escenarios a gran escala (alrededor de $782 \mathrm{~m} 3$ ). En casos intermedios debiera testearse en primer lugar el tamaño de celda de $5 \mathrm{~cm}$ y evaluar la velocidad de resolución de la simulación y en función del mismo definir si se desea continuar o acortar el tiempo de cómputo permitiendo un ligero error en la simulación.

\section{AGRADECIMIENTOS}

Los autores quieren agradecer al CSN (Consejo de Seguridad Nuclear) por la colaboración y cofinanciación del Proyecto «Simulación de Incendios en Centrales Nucleares».

\section{REFERENCIAS}

(1) Pryor, A.J. (1977) The Browns Ferry nuclear plant fire. Boston: SFPE, 77-2.

(2) NFPA 805: Performance-Based Standard for Fire Protection for Light Water Reactor Electric Generating Plants. National Fire Protection Association, 2015 Edition.

(3) Instrucción IS-30, sobre requisitos del programa de protección contra incendios en centrales nucleares. Consejo de Seguridad Nuclear, CSN, 2011.

(4) Rey, E., Aguayo, F., Peralta, M. ${ }^{a}$ E., Lama, J. R., Ávila, M. ${ }^{a}$ J. (2015). Integración de métodos escalares y vectoriales en la evaluación del riesgo de incendio en el ciclo de vida de una construcción. Informes de la Construcción, 67(539), doi: http://dx.doi.org/10.3989/ic.14.154

(5) McGrattan, K., y otros. (2016). Fire Dynamics Simulator (Version 6) Technical Reference Guide - Volume 1: Mathematical Model, NIST Special Publication 1018-1, Maryland, USA.

(6) United States Nuclear Regulatory Commission (U.S. NRC), Office of Nuclear Regulatory Research (RES), and Electric Power Research Institute (EPRI). (2007) NUREG-1824: Verification and Validation of Selected Fire Models for Nuclear Power Plant Applications, Volume 1: Fire Main report. Washington, DC, and Palo Alto, CA, USA

(7) United States Nuclear Regulatory Commission (U.S. NRC). (1999) NUREG-1758: Evaluation of Fire Models for Nuclear Power Plant Applications: Cable Tray Fires. Washington DC.

(8) Capote, J.A., Alvear, D., Abreu, O.V., Lázaro, M., Espina, P. (2008). Influencia del Modelo de Turbulencia y del Refinamiento de la Discretización Espacial en la Exactitud de las Simulaciones Computacionales de Incendios, Revista Internacional de Métodos Numéricos para Cálculo y Diseño en Ingeniería, 24(3): 227-245.

(9) Lázaro, M. (2008) Influencia de la Discretización Espacial en la Exactitud del Modelado de Fluidodinámica Computacional de Incendios, Tesis Doctoral, Universidad de Cantabria.

(10) Rein, G., Torero, J.L., y otros. (2009). Round-robin study of a priori modelling predictions of the Dalmarnock Fire Test One. Fire Safety Journal, 44(4): 590-602, doi: http://dx.doi.org/10.1016/j.firesaf.2008.12.008

(11) United States Nuclear Regulatory Commission (U.S. NRC), Office of Nuclear Regulatory Research (RES), and Electric Power Research Institute (EPRI). (2007) NUREG-1824: Verification and Validation of Selected Fire Models for Nuclear Power Plant Applications, Volume 7: Fire Dynamics Simulator (FDS). Washington, DC, and Palo Alto, CA, USA. 
(12) United States Nuclear Regulatory Commission (U.S. NRC), Office of Nuclear Regulatory Research (RES), and Electric Power Research Institute (EPRI). (2006). NUREG/CR-6931: Cable Response to Live Fire (CAROLFIRE), Sandia National Laboratories. Albuquerque, New México.

(13) McGrattan, K., y otros. (2016). Fire Dynamics Simulator (Version6) User Guide. NIST Special Publication 1019, Maryland, USA.

(14) United States Nuclear Regulatory Commission (U.S. NRC), Office of Nuclear Regulatory Research (RES), and Electric Power Research Institute (EPRI). (2012). NUREG-1934: Nuclear Power Plant Fire Modeling Analysis Guidelines (NPP FIRE MAG). Final Report, Washington, DC, and Palo Alto, CA, USA. 